\title{
Preliminary Results on Clinico- pathological and Immunohistochemical Features of Malignant Melanomas
}

\author{
Ciortea Carmen Diana1, Gurzu Simona², Zdrob Simona Maria², Jung ${ }^{2}$ \\ ${ }^{1}$ Department of Dermatovenerology, County Emergency Clinical Hospital, Tîrgu Mureș, Romania \\ 2 Department of Pathology, University of Medicine and Pharmacy, Tîrgu Mureș, Romania
}

Objective: To perform a retrospective clinico-pathological and immunohistochemical analysis of malignant melanomas. Methods: One-hundred sixty consecutive cases diagnosed in the Department of Pathology of the County Emergency Clinical Hospital of Tîrgu Mureș, Romania, between January 2000 - December 2009, were used. Of these, 47 cases were melanoma metastases that were excluded from the study; this research was focused on the study of 113 primary melanomas (PMs). In 20 cases of PMs (11 cutaneous, 5 choroidal, and 4 melanomas of the anal canal) immunohistochemical stains were performed using the antibodies p53, Ki67, ER (estrogen receptor), CD8 and CD31.

Results: Cutaneous PMs prevailed (83\%) followed by choroidal (11\%), digestive tract (4\%) and nasal mucosa PMs (2\%). Independent of their location, PMs predominantly affected females (58\%) over age 50. Regarding cutaneous cases, lower limbs (49\%) were more affected, followed by the head and neck (18\%) and the trunk (18\%). We noted negativity or low p53-nuclear positivity in cutaneous and digestive tract PMs; p53 was overexpressed in choroidal melanomas. On the other hand, although the proliferative index Ki67 was also quite low in cutaneous PMs, its percentage increased in choroidal and digestive tract PMs. CD8 was positive in cutaneous PMs and negative in choroidal and digestive tract cases. While in cutaneous PMs we noted a predominated angiogenesis by intussusception, in digestive tract melanomas there was a predominance of sprouting and pseudoglomeruloid mechanisms.

Conclusions: Choroidal PMs display the highest p53 and Ki-67 expression, compared to cutaneous MMs. Tumor angiogenesis seems to present a specific pathway in cutaneous PMS compared to the gastrointestinal cases.

Keywords: malignant melanoma, CD31, p53, Ki67, angiogenesis

Received: 10 May 2012

\section{Introduction}

In most of the cases, malignant melanomas (MMs) affect the skin, but they can be also located in the choroid, nasal cavity, gastrointestinal and even the urogenital tract [1-7]. Despite the newest targeted drugs, such as monoclonal BRAF-inhibitors, MM remains the most deadly malignant tumor of the skin, with an increasing incidence [8]. It predominantly affects Caucasians, being uncommon in black people and Asians. Several risk factors were identified, such as genetic predisposition, ultraviolet irradiation, family history, dysplastic nevi $[9,10]$. The diagnosis of non-cutaneous MMs is difficult; it requires a correct differentiation from metastases, undifferentiated carcinomas and other mesenchymal tumors.

The aim of this paper is to present clinico-pathological data of MMs diagnosed in our laboratory and to present the preliminary results regarding some immunohistochemical examinations. The mechanisms of angiogenesis, the process of formation of new vessels from pre-existing blood vessels, were also analyzed.

\section{Methods}

Surgical specimens from 160 consecutive MM cases, diagnosed in the Department of Pathology of the County Emergency Clinical Hospital of Tîrgu Mureş, Romania, between January 2000 - December 2009, were enrolled in the study. Of these, 47 were metastases, which were eliminated from the study, our research focusing on the remaining 113 cases of primary melanomas (PMs).

For each of the cases, the patient's gender and age, tumor location and the recurrence rate were analyzed. For cutaneous PMs, the histological type, Breslow index and tumor stage were also examined.

The statistical analysis was performed using the GraphPad InStat 3 programme. A significant association was considered when $\mathrm{p}<0.05$, with a $95 \%$ confidence interval.

\section{Immunohistochemical staining}

The immunohistochemical stains were performed in 20 randomly selected cases of PMs (11 cutaneous, 5 choroidal and 4 melanomas of the anal canal). To confirm the diagnosis, the antibodies anti-HMB45 (clone MS364, dilution 1:50) and S-100 protein (polyclonal rabbit, dilution 1:6000) from LabVision were used. We also performed immunohistochemical stains with a panel of five antibodies provided by LabVision: p53 (clone DO-7, dilution 


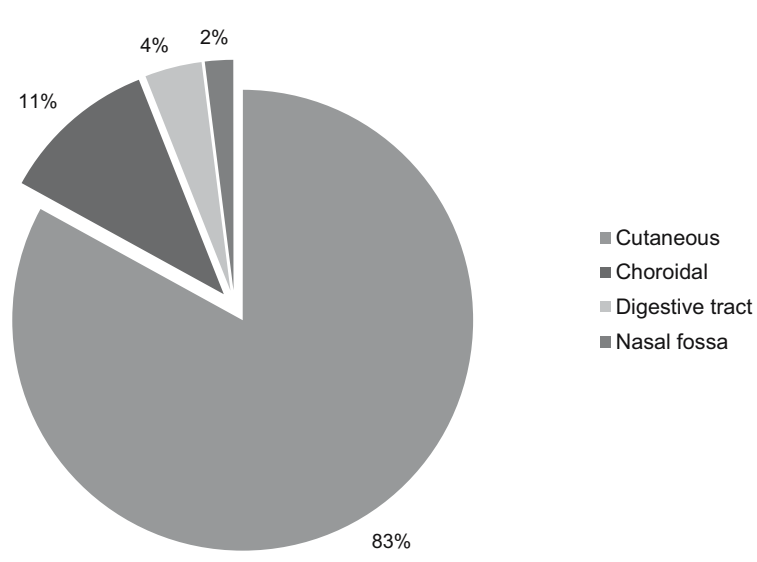

Fig. 1. Distribution of melanomas by location

1:50), Ki67 (clone Ki-S5, dilution 1:200), Estrogen Receptor (clone 1D5, dilution 1:100), CD8 (clone C8/144B, dilution 1:50) and CD31 (clone JC70A, dilution 1:25).

The immunohistochemical stains were performed on paraffin-embedded tissues, using the UltraVision System (LabVision). The heat-induced antigen retrieval was performed in high-retrieval solution for CD31 and citrate solution for the other antibodies. The slides were developed using diaminobenzidine (DAB; Dako) and counterstained with Mayer`s hematoxylin. For negative controls, incubation was performed with omission of the specific antibodies.

\section{Interpretation of immunohistochemical stains}

For anti-p53, Ki67 and Estrogen Receptor (ER) antibodies, the average percentage of the positive nuclei was quantified in the tumor cells, a cut-off value of $10 \%$ being considered.

For anti-CD8 antibodies we determined the number of intratumoral T-cells (intratumoral lymphocytic infiltrate), at a $200 \times$ magnification.

Angiogenesis was quantified using anti-CD31 antibodies and a Nikon E800 microscope, equipped with a video camera. To determine microvessel density (MVD), we identified the intratumoral areas with the most intense vascular density ("hot spot areas") and captured digital images from these regions, at 400 high power fields. In these areas, we counted the number of neoformed vessels (MVD). Both individual endothelial cells and vessels with lumen were taken into account. The ulcerated zones and areas with a high amount of inflammatory infiltrate were eliminated. The mechanisms of angiogenesis were analyzed, based on the arhitecture of the tumor cells. Endothelial sprouting, vessel co-option, intussusception, pseudoglomeruloid pattern and vasculogenic mimicry were explored, based on the previously well-known classifications [11].

\section{Results}

\section{Clinicopathological data}

Of 113 patients with PMs, 58\% were females and 42\% males. The average age was $60.59 \pm 14.32$ years. Female patients had an average age of $60.11 \pm 15.03$ years and males $61.23 \pm 13.39$ years, without a statistically significant difference $(p=0.62)$. The incidence of melanomas increased, in both genders, over the age of 50 years.

Most of the cases were cutaneous PMs (94 cases) followed by choroidal (12 cases), anal canal (5 PMs) and nasal mucosa PMs (2 cases) (Figure 1).

Both melanomas of the nasal mucosa were diagnosed in females and of the 12 choroidal melanomas, 9 cases were diagnosed in male patients (19.14\% of all melanomas), and 3 in female patients ( $4.54 \%$ of all melanomas). The proportion of cutaneous and gastrointestinal melanomas had a relatively equal distribution among genders.

\section{Particularities of cutaneous PMs}

Independently by the patient's gender, the lower limbs (49\%) were more affected, followed by the head and neck $(18 \%)$, trunk (18\%) and the upper limbs (15\%). In females, PMs occurred mostly on the lower limbs (49\%) followed by the trunk (21\%); in males, the trunk (35\%) was
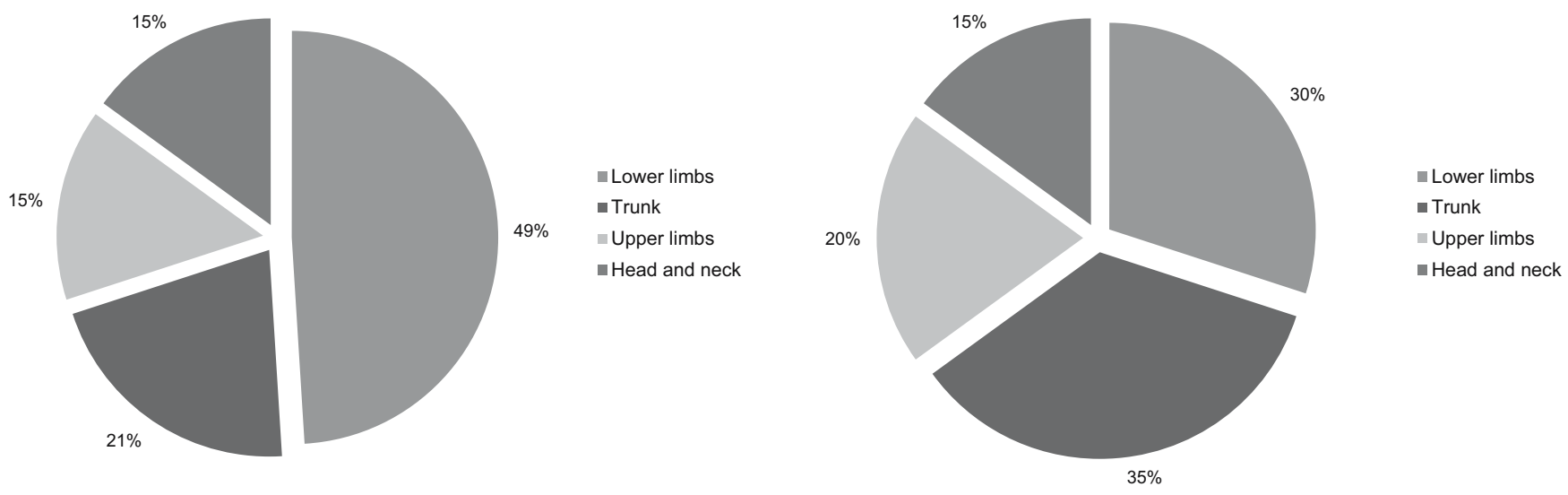

Fig. 2. Localization of cutaneous melanomas by gender: females (left) and males (right) 


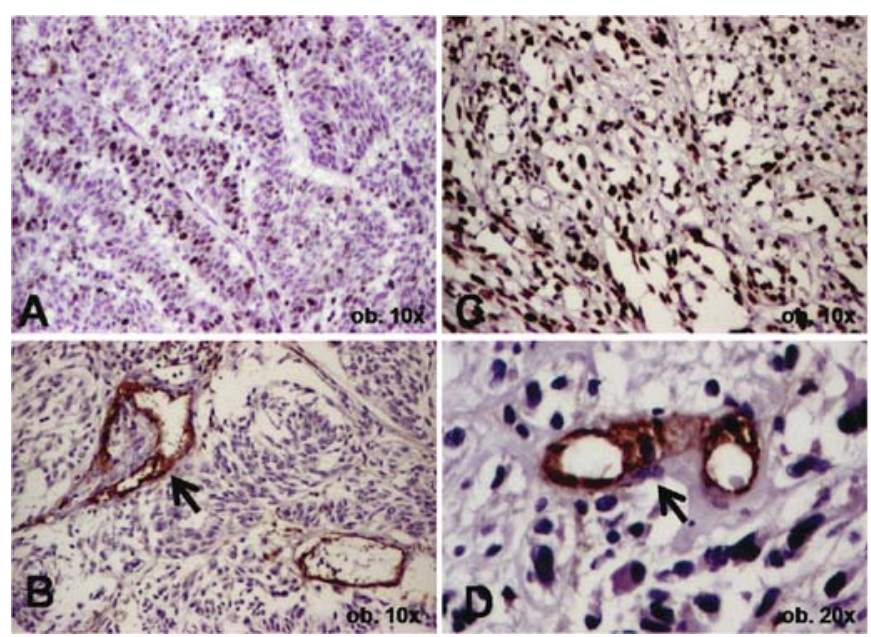

Fig. 3. The immunohistochemical findings in primary malignant melanomas (PMs). A low Ki67 index (A) and intusussception angiogenesis $(\mathrm{B})$ are characteristic for cutaneous $\mathrm{PM}$; PMs of the anal canal display a higher Ki67 index (C) and a pseudoglomeruloid angiogenic pattern (D)

the most common location, followed by the lower limbs (30\%) (Figure 2).

More than $2 / 3$ s of the 94 cutaneous PMs were nodular (78\%), followed by superficial (16\%) and lentiginos-type melanoma (6\%). Most of these tumors presented invasion of the reticular dermis (49\%), regardless of location, age and gender. The microscopic type of melanomas was not correlated with the depth of invasion $(\mathrm{p}=0.45)$, but nodular tumors had a larger diameter than superficial PMs $(\mathrm{p}=$ 0.015).

The average diameter was $5.96 \pm 2.34 \mathrm{~mm}$ (ranging between $0.4-30 \mathrm{~mm}$ ). In females the average diameter was $5.11 \pm 1.54 \mathrm{~mm}$; in males, it was slightly higher $7.65 \pm$ $3.21 \mathrm{~mm}$, with a difference at the limit of statistical significance $(p=0.05)$. The tumor size was not correlated with the patient's age or the localization of cutaneous PMs $(\mathrm{p}=0.52)$. However, all PMs larger than $15 \mathrm{~mm}$ presented invasion of the hypodermis and subcutaneous tissues $(\mathrm{p}$ $<0.01$ ).

In the analyzed material, the Breslow index increased in parallel with the depth of invasion $(p=0.002)$. In terms of Clark level, statistical differences regarded the pT4 and pT1 stages $(p=0.001)$. Thus, all melanomas with Clark level V were diagnosed in stage pT4 and invasion limited to the epidermis was observed only in melanomas $\leq 1 \mathrm{~mm}$, namely those diagnosed in stage pT1. Regarding pTNM staging, most of the cutaneous PMs were diagnosed in stage pT4 (53\%) followed by stages pT3 (21\%), pT1 $(10 \%)$ and pT2 (20\%).

\section{Correlation of microscopic aspects with recurrence/ metastasis rate}

From 113 PMs, regardless of location, 14 (12.38\%) presented recurrence and/or metastasis. Out of 14 patients, 13 were females and one male. In 12 cases, the primary tumor was cutaneous-located, the metastases being identified in the liver and brain. One esophageal PM presented a cutaneous metastasis, and a nasal mucosa PM recurred.

\section{The immunohistochemical features}

\section{$E R, p 53$ and Ki67 expression}

None of the 20 cases expressed ER.

In cutaneous PMs, we noted negativity or low expression of the p53 protein $(<20 \%)$, regardless of tumor stage, presence/absence of recurrences/metastasis. PMs of the digestive tract did not express $\mathrm{p} 53$. In choroidal melanomas, p53 was overexpressed, being identified in more than 50\% of the nuclei of tumor cells.

The proliferative index Ki67 was low in cutaneous PMs, in most of cases being expressed in $<5-10 \%$ of the tumor cell nuclei. In choroidal melanomas, the average index of Ki67 positivity was about 25\%. In PMs of the digestive tract, its average value was $75 \%$ (Figure 3, Table I). There were no correlations between p53 and Ki67 expression.

\section{Angiogenesis and CD8 expression in cutaneous PMs}

The average number of intratumoral vessels, marked by CD31 (MVD), was $3.21 \pm 1.23$, independent of the microscopic type, Breslow index, Clark level or other clinico-pathological factors. Angiogenesis by intussusception was the predominant mechanism observed in the $10 \mathrm{PMs}$, most of the vessels being large, with well-defined lumen (Figure 3).

An interesting aspect was observed regarding the correlation between CD31 and TIL value, quantified with

Table I. Clinico-pathological aspects of primary malignant melanomas (PMs) correlated with the p53 and Ki67 expression

\begin{tabular}{|c|c|c|c|c|c|}
\hline \multicolumn{2}{|c|}{ Parameter } & \multirow{2}{*}{$\begin{array}{c}\begin{array}{c}\text { Cutaneous PMs } \\
(n=94)\end{array} \\
36(38.30 \%)\end{array}$} & \multirow{2}{*}{$\begin{array}{c}\begin{array}{c}\text { Choroidal PMs } \\
(\mathrm{n}=12)\end{array} \\
9(75 \%)\end{array}$} & \multirow{2}{*}{$\begin{array}{l}\text { PMs of the anal canal } \\
\qquad(n=5)\end{array}$} & \multirow{2}{*}{$\begin{array}{l}\text { PMs of the nasal cavity } \\
\qquad(\mathrm{n}=2)\end{array}$} \\
\hline Gender & Male $(n=47)$ & & & & \\
\hline & Female $(n=66)$ & $58(61.70 \%)$ & $3(25 \%)$ & $3(60 \%)$ & $2(100 \%)$ \\
\hline \multicolumn{2}{|c|}{ Average age (years) } & $58.23 \pm 14.34$ & $62.34 \pm 11.57$ & $59.69 \pm 15.61$ & 61 \\
\hline \multirow[t]{3}{*}{ p53 } & negative & 30 (31.91\%) & - & $5(100 \%)$ & NA \\
\hline & $<50 \%$ & $64(68.09 \%)$ & - & - & NA \\
\hline & $>50 \%$ & - & $12(100 \%)$ & - & NA \\
\hline \multirow[t]{3}{*}{ Ki67 } & negative & $73(77.66 \%)$ & $4(33.33 \%)$ & - & NA \\
\hline & $<50 \%$ & $21(22.34 \%)$ & $8(66.67 \%)$ & $1(20 \%)$ & NA \\
\hline & $>50 \%$ & - & - & $4(80 \%)$ & NA \\
\hline
\end{tabular}


CD8. In most of the cases, 2-3 CD8-positive TILs were identified at the $200 \times$ magnification. In these cases, a few mature vessels (1-2/field, HPF) with well-defined lumen were observed. On the other hand, in those cases that displayed a higher number of TILs (over six per microscopic field), neoformed vessels were also present in a significant number, with the predominance of isolated endothelial cells or well-defined vessels with a thin wall and very small lumen.

\section{Angiogenesis and CD8 expression in choroidal PMs}

Despite of a high p53 index in choroidal melanomas, there were no intratumoral lymphocytes (CD8 was negative) and angiogenesis was extremely low, only one or two fully mature, large vessels were rarely observed.

\section{Angiogenesis and $C D 8$ expression in PMs of the digestive tract (anal canal) \\ In all four cases located in the anal canal, CD8 was nega- tive, but angiogenesis was intense, with the predominance of isolated endothelial cells or well-defined vessels with thin wall and very small lumen. Sprouting angiogenesis and pseudo-glomeruloid pattern predominated (Figure 3).}

\section{Discussion}

In the light of our results, which showed a slight predominance of females compared to males $(\mathrm{M}: \mathrm{F}=1: 1.2)$ and the predominance of lower limb cutaneous PMs in females, and trunk PMs in males, respectively, there are authors who revealed the importance of sunlight exposure in the pathogensis of PMs of the skin [12]. Apart from the skin and choroidal plexus, primary malignant melanomas are rare at the other sites, these being reported in the literature mainly as case presentations $[3,5,7]$.

The prognosis of cutaneous PM depends on the microscopic type of tumor (nodular vs. superficial), Breslow index, presence of ulceration, the status of sentinel lymph nodes and tumor stage [9,13-16]. In our study, an average tumor diameter of $5.96 \mathrm{~mm}$ was described, all tumors over $15 \mathrm{~mm}$ being diagnosed in the pT4 stage; therefore, pTNM stage remains the most important prognostic criterion.

There are several recent studies that highlight the importance of various antibodies and molecular examinations to predict the prognosis of PMs. One of this marker, undetected in our cases, is ER $\beta$. There is a supposition about its role in the progression of cutaneous PM; some authors proved a progressively decreasing expression of ER at the same time with the increase of Breslow index and tumor stage, respectively $[17,18]$.

Both p53 and Ki67 were poorly expressed in cutaneous PMs, but choroidal PMs showed intense p53 nuclear positivity. Nuclear Ki67 proliferation index was increased in PMs located in the anal canal, but it was not correlated with p53 expression in any of the locations. However, overexpression of Ki67 is associated with lower survival rates [19].
An important topic in the field of melanoma research is related to angiogenesis; it can be inhibited using antiangiogenic substances, but the results are below expectations; a proper inhibition might make tumors highly susceptible to other chemotherapics [20]. The mechanisms of angiogenesis still remain unelucidated. Although previous studies revealed that the vessels co-option is the most commonly involved mechanism [21], intussusception was the angiogenic pattern observed in our material, in cutaneous PMs; a pseudo-glomeruloid angiogenesis was marked out by the PMs of the anal canal. No other studies showed the presence of these possible mechanisms in melanoma pathogenesis.

\section{Conclusions}

Diverse angiogenic mechanisms and features of agressivity are present in cutaneous melanomas, compared to choroidal and gastrointestinal primary melanomas. Intussusception angiogenesis seems to predominate in cutaneous melanomas, but the pseudo-glomeruloid pattern is rather characteristic for melanomas of the anal canal. A proper understanding of the mechanisms of angiogenesis involved in the pathogenesis of melanoma should be explored in further studies, as a possible cause of resistance to antiangiogenic therapy.

\section{Acknowledgement}

This paper is partly supported by the "For women in science" National Fellowship and the Sectoral Operational Programme Human Resources Development (SOP HRD), financed from the European Social Fund and by the Romanian Government under the contract number POSDRU 80641.

\section{References}

1. Ivanisević P, Bojić L, Tomić S, et al. Clinico-epidemiological analysis of choroidal melanoma in Split Area, Croatia. Acta Med Croatica. 2011;65:257-261.

2. Uysal IÖ, Misir M, Polat K, et al. Primary malignant melanoma of the nasal cavity. J Craniofac Surg. 2012;23:2-5.

3. Yamamura K, Kondo K, Moritani S. Primary malignant melanoma of the stomach: report of a case. Surg Today. 2012; 42:195-199.

4. Díaz-Sánchez A, Lara MÁ, Ortega P, et al. Colorectal melanoma: review of two distinct forms of presentation. Gastroenterol Hepatol. 2011; 34:83-88.

5. Ramos JA, Ramos WE, Ramos CV. Melanoma of the female urethra. Indian J Urol. 2011; 27:448-450.

6. Doublali M, Chouaib A, Khallouk A, et al. Primary malignant melanoma of prostate. Urol Ann. 2010;2:76-77.

7. Inoue M, Ishioka J, Kageyama $\mathrm{Y}$, Fukuda H, Higashi $\mathrm{Y}$. Primary malignant melanoma of the male urethra: a case report. Hinyokika Kiyo. 2008;54:305-308.

8. Sosman JA, Kim KB, Schuchter L, et al. Survival in BRAF V600mutant advanced melanoma treated with vemurafenib. $\mathrm{N}$ Engl $\mathrm{J}$ Med. 2012;366:707-714.

9. Gandini S, Sera F, Cattaruzza MS, et al. Meta-analysis of risk factors for cutaneous melanoma: III. Family history, actinic damage and phenotypic factors. Eur J Cancer. 2005;41:2040-2059.

10. Anger M, Friedhofer H, Fukutaki MF, Ferreira MC, Landman G. Primary cutaneous melanoma: an 18-year study. Clinics (Sao Paulo). 2010;65:257263.

11. Döme B, Hendrix JC, Paku S et al. Biological perspectives. Alternative vascularization mechanism in cancer. Pathology and therapeutic implications. Am J Pathol 2007;170:1-15. 
12. Battisti R, Nunes DH, Weber AL, Schweitzer LC, Sgrot I. Evaluation of the epidemiological profile and the mortality rate of the patients with primary cutaneous melanoma in Florianopolis - SC, Brazil. An Bras Dermatol. 2009;84:335-342.

13. Lugowska I, Kowalska M, Zdzienicki M, et al. The prognostic role of clinical factors, VEGF, IL-8 and STNF-R1 in cutaneous melanomas at locoregional stage. Pol Merkur Lekarski. 2012;32:22-27.

14. Garbe C, Hauschild A, Volkenandt M, et al. Evidence and interdisciplinary consense-based German guidelines: diagnosis and surveillance of melanoma. Melanoma Res. 2007;17:393-399.

15. Eggermont AM, Spatz A, Lazar V, Robert C. Is ulceration in cutaneous melanoma just a prognostic and predictive factor or is ulcerated melanoma a distinct biologic entity? Curr Opin Oncol. 2012;24:137-140.

16. Kovacević P, Visnjić M, Vlajković M, Kovacević T, Visnjić A. Sentinel node biopsy for skin melanoma. Vojnosanit Pregl. 2009;66:657-662.
17. Ohata C, Tadokoro T, Itami S. Expression of estrogen receptor beta in normal skin, melanocytic nevi and malignant melanomas. J Dermatol. 2008;35:215-221.

18. de Giorgi V, Gori A, Grazzini M, et al. Estrogens, estrogen receptors and melanoma. Expert Rev Anticancer Ther. 2011;11:739-747.

19. Vaisanen A, Kuvaja P, Kallioinen M, et al. A prognostic index in skin melanoma through the combination of matrix metalloproteinase-2, Ki67, and p53. Hum Pathol. 2011;42:1103-1111.

20. Helfrich I, Schadendorf D. Blood vessel maturation, vascular phenotype and angiogenic potential in malignant melanoma: One step forward for overcoming anti-angiogenic drug resistance? Mol Oncol. 2011;5:137149.

21. Mahabeleshwar GH, Byzova TV. Angiogenesis in Melanoma. Semin Oncol. 2007;34:555-565. 